\title{
ANALYTICITY OF THE INTERFACE OF THE POROUS MEDIA EQUATION AFTER THE WAITING TIME
}

\author{
SIGURD ANGENENT
}

(Communicated by Walter Littman)

\begin{abstract}
We prove that the free boundary of a solution of the porous media equation, with compactly supported initial data, is real analytic after the waiting time. The proof uses the maximal regularity theory of Da Prato and Grisvard.
\end{abstract}

1. Introduction. Consider the Cauchy problem for the porous media equation,

$$
\begin{cases}u_{t}=\left(u^{m}\right)_{x x}, & x \in \mathbf{R}, t>0, \\ u(x, 0)=u_{0}(x), & x \in \mathbf{R} .\end{cases}
$$

We assume that $u_{0}$ is continuous, nonnegative and that $\left\{x \mid u_{0}(x)>0\right\}$ is a bounded interval.

It is known that problem (1) has a unique weak solution, which in general is not a classical solution. This solution is continuous and nonnegative on $[0, \infty) \times \mathbf{R}$. that

For fixed $t>0, u(t, \cdot)$ has compact support and there are functions $\zeta_{ \pm}(t)$ such

$$
\{x \mid u(t, x)>0\}=\left(\varsigma_{-}(t), \varsigma_{+}(t)\right) .
$$

These functions $\varsigma_{ \pm}(t)$ are known to be locally Lipschitz functions of $t>0$, such that $\varsigma_{+}(t)$ and $-\varsigma_{-}(t)$ are nondecreasing. The curves $x=\varsigma_{ \pm}(t)$ are called the free boundaries of the solution $u(t, x)$.

There exist numbers $t_{ \pm} \geq 0$ such that $\varsigma_{ \pm}(t)$ is constant for $t \leq t_{ \pm}$and strictly monotone for $t \geq t_{ \pm}$. These numbers $t_{ \pm}$are the so-called waiting times for $\zeta_{+}$and $S_{-}$.

We refer to the survey article of Aronson [3] for more references and background information on the free boundaries $\zeta_{ \pm}$.

In [5] Caffarelli and Friedman have shown that on $\varsigma_{ \pm}(t)$ is a $C^{1}$ function of $\left(t_{ \pm}, \infty\right)$.

Recently Aronson and Vazquez [4], and Höllig and Kreiss [6] have improved this result by showing that $\zeta_{ \pm}$is actually $C^{\infty}$ on $\left(t_{ \pm}, \infty\right)$.

Our main result is the following.

THEOREM 1. On $\left(\max \left(t_{+}, t_{-}\right), \infty\right) \varsigma_{+}$and $\varsigma_{-}$are real analytic functions.

The proof consists of three steps. First we write problem (1) as an abstract parabolic initial value problem in the sense of Da Prato and Grisvard [7]. The theory in [7] then allows us to conclude that problem (1) generates a local semiflow in a suitable Banach space. Thus one obtains a local existence result. An easy

Received by the editors October 26, 1985 and, in revised form, October 14, 1986.

1980 Mathematics Subject Classification (1985 Revision). Primary 35K65; Secondary 58D07. 
argument involving the analytic dependence of solutions on parameters allows one to conclude that these local solutions are real analytic.

In the second step one shows that the local semiflow is actually a global semiflow. This amounts to establishing a priori estimates on a Hölder norm of $u^{m-1}\left(u^{m-1}\right)_{x x}$ in the interval $\left[\zeta_{-}(t), \zeta_{+}(t)\right]$.

These two steps allow us to prove Theorem 1 with the extra assumption that for some $t$ after the waiting time $u(t, \cdot)$ has a certain amount of smoothness (to be specified below).

The final step of the proof then invokes a recent estimate of Aronson and Vazquez (namely that $\left(u^{m-1}\right)_{x x}$ is bounded) in order to show that $u(t, \cdot)$ has the required smoothness for arbitrary small $t>\max \left(t_{+}, t_{-}\right)$, so that we can conclude analyticity of $\varsigma_{ \pm}(t)$ for $t>\max \left(t_{+}, t_{-}\right)$.

2. Local existence and analyticity. It will be convenient to work with $v=m u^{m-1}$ instead of $u$. We have

$$
v_{t}=v v_{x x}+\frac{1}{m-1}\left(v_{x}\right)^{2}
$$

Next we consider the function

$$
V(t, \xi)=v\left(t, \frac{\zeta_{+}+\zeta_{-}}{2}+\frac{\zeta_{-}-\zeta_{+}}{2} \cos \xi\right) \quad(\xi \in \mathbf{R}, t \geq 0) .
$$

A direct computation shows that $V$ formally satisfies

$$
\begin{aligned}
V_{t}= & \left(\frac{2}{\zeta_{+}-\zeta_{-}}\right)^{2}\left[\frac{V}{\sin ^{2} \xi}\left(V_{\xi \xi}-\cot \xi V_{\xi}\right)+\frac{1}{m-1}\left(\frac{V_{\xi}}{\sin \xi}\right)^{2}\right] \\
& +\frac{(1+\cos \xi) Z_{-}+(1-\cos \xi) Z_{+}}{\varsigma_{+}-\zeta_{-}} \cdot \frac{V_{\xi}}{\sin \xi}
\end{aligned}
$$

where $Z_{ \pm}(t)=s_{ \pm}^{\prime}(t)$

Although the transformation $x \rightarrow \xi$ has turned the relatively simple equation (2) into the more complicated (3) one should note the following:

(a) $V(t, \cdot)$ is a $2 \pi$ periodic even function.

(b) The free boundary $x=\varsigma_{+}\left(\zeta_{-}\right)$corresponds to $\xi=\pi+2 \pi \mathbf{Z}(0+2 \pi \mathbf{Z})$, and therefore has a fixed position in the new coordinate $\xi$.

(c) The transformation $\xi \rightarrow x(\xi)$ is regular at all $\xi \in \mathbf{R}$, except at $\xi \in \pi \mathbf{Z}$ (i.e. at the free boundary). At $\xi=0$ one has the following relations:

$$
V(t, \xi)=v_{x}\left(t, \zeta_{-}\right) \frac{\zeta_{+}-\zeta_{-}}{4} \cdot \xi^{2}+o\left(\xi^{2}\right)
$$

i.e.,

$$
\begin{aligned}
& V_{\xi}(t, 0)=0, \\
& \frac{V_{\xi}(t, \xi)}{\sin \xi}=\frac{\varsigma_{+}-\varsigma_{-}}{2} \cdot v_{x}\left(t, \zeta_{-}\right)+o(1), \\
& \frac{V(t, \xi)}{\sin ^{2} \xi}=\frac{\varsigma_{+}-\varsigma_{-}}{4} v_{x}\left(t, \zeta_{-}\right)+o(1),
\end{aligned}
$$

and at $\xi=\pi$ one obtains similar relations. 
(d) If $v_{x}\left(t, \varsigma_{ \pm}\right)$is nonzero, then the relations above imply that $V(t, \xi) / \sin ^{2} \xi$ is strictly positive on $\mathbf{R}$, so that (3) becomes uniformly parabolic.

So (3) is a nondegenerate parabolic equation. The price we have to pay for eliminating the degeneracy in (2) is the occurrence of singularities in first order terms (i.e. $\cot (\xi) \cdot V_{\xi}$, and $V_{\xi} / \sin \xi$ ) in the equations.

Equation (3) by itself is not enough to describe the time development of $v(t, x)$ : one needs equations for $\zeta_{+}^{\prime}$ and $\zeta_{-}^{\prime}$.

These may be derived from the condition $V_{t}(t, 0)=V_{t}(t, \pi)=0(t>0)$ and from (3). One obtains

$$
\zeta_{ \pm}^{\prime}(t)=Z_{ \pm}(t)=\frac{2}{m-1} \cdot \frac{V_{\xi \xi}\left(t, \xi_{ \pm}\right)}{\zeta_{+}-\zeta_{-}}
$$

where $\xi_{+}=\pi$ and $\xi_{-}=0$.

If we now regard $V, \zeta_{+}$and $\zeta_{-}$as independent variables then (3) and (4) give us an initial value problem for $\left(V, \varsigma_{+}, \varsigma_{-}\right)$. In order to state our local existence result we have to introduce a number of Banach spaces.

For $0<\theta<1$ define

$$
\begin{gathered}
E_{0, \theta}=\left\{V \in h^{\theta}(\mathbf{R}): V(\xi) \equiv V(\xi+2 \pi) \equiv V(-\xi) \text { and } V(0)=V(\pi)=0\right\} \\
E_{1, \theta}=h^{2+\theta}(\mathbf{R}) \cap E_{0, \theta} .
\end{gathered}
$$

Here $h^{s}(\mathbf{R})$ denotes the little Hölder space of exponent $s \in \mathbf{R} \backslash \mathbf{Z}$.

Furthermore let $X_{0, \theta}$ and $X_{1, \theta}$ be defined by

$$
X_{k, \theta}=E_{k, \theta} \oplus \mathbf{R}^{2} \quad(k=0,1),
$$

and consider the open subset $O_{\theta}$ of $X_{1, \theta}$ given by

$$
\begin{aligned}
O_{\theta}=\left\{\left(V, \varsigma_{-}, \varsigma_{+}\right) \in X_{1, \theta} \mid \varsigma_{-}<\varsigma_{+}, V(\xi)>0\right. & \text { for } 0<\xi<\pi \\
& \text { and } \left.V_{\xi \xi}(0), V_{\xi \xi}(\pi)>0\right\} .
\end{aligned}
$$

We remind the reader that, due to the singularity of the transformation $\xi \rightarrow x$, one has $V_{\xi \xi}(0)=\frac{1}{2}\left(\varsigma_{+}-\varsigma_{-}\right) v_{x}\left(t, \varsigma_{-}\right)$, so that the condition $V_{\xi \xi}>0$ at $\xi=0, \pi$ is equivalent to $v_{x} \neq 0$ at $x=\varsigma_{ \pm}$.

The initial value problem (3), (4) may now be written as

$$
w^{\prime}(t)=F(w(t))
$$

where $F: O_{\theta} \rightarrow X_{0, \theta}$ is given by

$$
F\left(V, \varsigma_{+}, \varsigma_{-}\right)=\left(V_{t}, Z_{+}, Z_{-}\right)
$$

In [2] we prove the following result:

THEOREM 2. $F: O_{\theta} \rightarrow X_{0, \theta}$ is a real analytic map. For each $w \in O_{\theta}$ the Fréchet derivative $d F(w)$, seen as an unbounded operator in $X_{0, \sigma}$ with domain $X_{1, \sigma}(0<\sigma \leq \theta)$, is the generator of an analytic semigroup.

The proof of this theorem is rather long, and the larger part of the proof is devoted to the problem of showing that $d F(w)$ generates an analytic semigroup. Using various perturbation theorems this problem may be reduced to that of showing that

$$
A=a(x)\left(\frac{d}{d x}\right)^{2}+\frac{b(x)}{x} \frac{d}{d x} \quad(0<x<\infty)
$$


with $0<a, a^{-1}, b \in h^{\theta}([0, \infty))$, generates an analytic semigroup in $h^{\theta}([0, \infty))$ if one takes

$$
\operatorname{dom}(A)=\left\{u \in h^{2+\theta}([0, \infty)) \mid u^{\prime}(0)=0\right\} .
$$

This is done in [2a] by explicitly constructing the resolvent of $A$.

Given Theorem 2 we can directly apply the theory of Da Prato and Grisvard [7]. We get the following

THEOREM 3. $F: O_{\theta} \rightarrow X_{0, \theta}$ generates a local semiflow on $O_{\theta}$.

Although they do not state this explicitly it follows at once from Da Prato and Grisvard's theory [7], especially the proof of Theorem 4.1 in p. 356) that, if $F$ depends smoothly on a parameter $\lambda$ (in some Banach space), then the solution also depends smoothly on $\lambda$ (one uses the Implicit Function Theorem). In particular, if $F$ depends analytically on $\lambda$ then so does $w$ (in the $C\left([0, T] ; X_{1, \theta}\right)$ topology).

Since $w(\lambda t)$ (for $\lambda \in \mathbf{R}_{+}$) is a solution of $w^{\prime}(t)=\lambda F(w(t))$, and $(\lambda, w) \mapsto \lambda F(w)$ is a real analytic map from $\mathbf{R}_{+} \times O_{\theta}$ to $X_{0, \theta}, w(\lambda t) \in C\left([0, T] ; X_{1, \theta}\right)$ depends analytically on $\lambda$. Hence, for $t>0, w(t)$ is real analytic. In particular, $\zeta_{+}$and $\zeta_{-}$ are real analytic on $(0, T]$. This completes the first step of the proof of Theorem 1.

3. Global analyticity. For any $w_{0} \in O_{\theta}$ we have shown that there exists a maximal solution $w:\left[0, t_{\max }\right) \rightarrow O_{\theta}$ of (5). In this section we shall prove that $t_{\max }=+\infty$.

It is a standard result from the theory of local semiflows that $t_{\max }=+\infty$ if the following holds: for any finite $t \leq t_{\max }$ the set $\{w(s) \mid 0 \leq s<t\}$ is precompact in $O_{\theta}$. Since $O_{\theta}$ is an open subset of $X_{1, \theta}$ we therefore have to verify that the set $\{w(s) \mid 0 \leq s<t\}$ stays away from the boundary of $O_{\theta}$, and is precompact in the larger space $X_{1, \theta}$.

The orbit segment will certainly stay away from the boundary of $O_{\theta}$, since it is known that $\zeta_{-}(t)$ is always strictly less than $\varsigma_{+}(t), V(t, \xi)$ is strictly positive on $0<\xi<\pi$ and $V_{\xi \xi}(t, \xi)$ is strictly positive at $\xi=0$ and $\xi=\pi$ (here we use the fact that our solutions coincide with the weak solutions of the porous media equation).

To prove $t_{\max }=+\infty$ it therefore suffices to prove that $w(s)=\left(V(s, \cdot), \zeta_{-}(s)\right.$, $\left.\varsigma_{+}(s)\right)$ remains in a compact subset of $X_{1, \theta}$ as long as $s<t$ for any finite $t \leq t_{\max }$. Since the free boundaries $\zeta_{ \pm}(s)$ are bounded on bounded time intervals, this gives us the following criterion for global existence.

LEMMA. If, for any $t \leq t_{\max }$, the set $\{V(s, \cdot) \mid 0 \leq s<t\}$ is precompact in $h^{2+\theta}(\mathbf{R})$ then $t_{\max }=+\infty$.

To obtain this precompactness we shall derive on a priori bound on a Hölder norm of $V_{\xi \xi}(t, \cdot)$, higher than $h^{\theta}$. Since the solution is $C^{\infty}$ away from the boundary we can obtain such an a priori estimate through a local study of the equation near the boundary.

In order to do this we return to equation (2) and introduce new coordinates $(y, t)$ which are related to $(x, t)$ via

$$
v(x, t)=\frac{1}{2} y^{2} .
$$

Using the result of Cafferelli and Friedman [5], which says that $v_{x}$ is uniformly continuous up to the boundary, one easily verifies that for fixed $t$ the coordinates $\xi$ 
and $y$ are $C^{1}$ functions of each other, up to the boundary and uniformly in bounded $t$-intervals.

Therefore Hölder continuity of $V_{\xi \xi}(t, \cdot)$ with respect to $\xi$ is the same as Hölder continuity with respect to $y$. In what follows we shall derive an a priori bound on the Hölder norm of $V_{\xi \xi}(t, \cdot)$ with respect to $y$.

First we derive an equation for $v_{x}$, as a function of $y$ and $t$. Differentiating the relation $2 v=y^{2}$ gives

$$
\begin{aligned}
& p \cdot x_{y}=y \\
& q \cdot\left(x_{y}\right)^{2}+p \cdot x_{y y}=1, \\
& v_{t}+p \cdot x_{t}=0
\end{aligned}
$$

where $p=v_{x}$ and $q=v_{x x}$. Thus we obtain

$$
-x_{t}=\frac{1}{2} y p_{y}+\frac{1}{m-1} p .
$$

Using $p \cdot x_{y}=y$ this gives the following equation for $p$ :

$$
p_{t}=\frac{1}{2} p^{2}\left(p_{y y}+\frac{m+1}{m-1} \frac{p_{y}}{y}\right)
$$

which holds whenever $p \neq 0$. Now let $t_{0} \leq t_{\max }$ be a finite given number. It is known that $p$ is continuous up to the free boundary (Caffarelli and Friedman [5]) so that there is a small $\eta>0$ such that (6) holds on $0 \leq t \leq t_{0}, 0 \leq y \leq \eta$. On the curve $y=\eta$ the function $p(t, y)$ is $C^{\infty}$, say $p(t, \eta)=\phi(t)$. Then we have the following initial-boundary value problem

$$
\begin{cases}p_{t}=\frac{1}{2} p^{2}\left(p_{y y}+\frac{m+1}{m-1} \frac{p_{y}}{y}\right), & 0 \leq t \leq t_{0},|y| \leq \eta \\ p(t, \eta)=\phi(t), & 0 \leq t \leq t_{0} \\ p(t,-y)=p(t,+y), & |y| \leq \eta \\ p(0, y), & \text { given }\end{cases}
$$

This equation is quasilinear, so that we may use the existence and regularity theory of Amann [1], or Angenent [2b]. In order to do this however, we must get rid of the inhomogeneous boundary condition $p=\phi(t)$. Therefore we define a differential operator

$$
A=\left(\frac{d}{d y}\right)^{2}+\frac{m+1}{m-1} \frac{1}{y} \frac{d}{d y}
$$

and we choose a $C^{\infty}$ function $\psi(t, y)$ which satisfies

$$
\begin{aligned}
& \psi(t,-y)=\psi(t, y), \\
& \psi_{t}=\frac{1}{2} \psi^{2} A(\psi) \text { for } 0 \leq t \leq t_{0}, \text { at } y=\eta
\end{aligned}
$$

and

$$
\psi(t, \eta)=\phi(t), \quad 0 \leq t \leq t_{0}
$$

Such a function may be defined in terms of $\phi(t)$ and $\phi^{\prime}(t)$ along (e.g. for a suitable choice of $k \in \mathbf{R}$,

$$
\psi(t, y)=\phi(t)+k \frac{\phi^{\prime}(t)}{\phi(t)^{2}}\left(1-\left(\frac{y}{\eta}\right)^{2}\right)^{2}
$$

is such a function). 
Next we write $p=P+\psi$, and we obtain the following equations for $P$ :

$$
\left\{\begin{array}{l}
P_{t}=\frac{1}{2}(P+\psi)^{2} A(P)+\frac{1}{2}(P+\psi)^{2} A(\psi)-\psi_{t}, \\
P(t, \eta)=0 \\
P(t,-y)=P(t, y) \\
P(t, 0) \text { given. }
\end{array}\right.
$$

This equation can be written as

$$
\left\{\begin{array}{l}
P_{t}=L(P, t) P+f(P, t) \\
P(0) \text { given }
\end{array}\right.
$$

where $L(P, t)$ is the differential operator given by $\frac{1}{2}(P+\psi)^{2} \cdot A$. This operator works in the space

$$
E_{0, \theta}=\left\{P \in h^{\theta}([-\eta,+\eta]) \mid P(-y)=P(y), P(\eta)=0\right\}
$$

and has domain

$$
E_{1, \theta}=\left\{P \in h^{2+\theta}([\eta, \eta]) \cap E_{0, \theta} \mid(A P)(\eta)=0\right\} .
$$

The mapping $(P, t) \mapsto f(P, t)=\frac{1}{2}(P+\psi)^{2} A(\psi)-\psi_{t}$ is well defined from $E_{0, \theta} \times\left[0, t_{0}\right]$ to $E_{0, \theta}$. In [2a] we prove the following

LEMMA. For $t \in\left[0, t_{0}\right]$ and $P \in E_{0, \theta}$ such that $p^{2}=(P+\psi)^{2}$ is strictly positive the operator $L(P, t)$ generates an analytic semigroup in $E_{0, \sigma}(0<\sigma \leq \theta)$.

The theory in [1 or $\mathbf{2 b}$ ] then implies the following local existence theorem.

THEOREM. If $p(0, y)$ belongs to $h^{\theta}([0, \eta])$ then problem (7) has a local solution. If this local solution remains bounded in $h^{\theta}$ then it is defined for $0 \leq t \leq t_{0}$.

The solutions produced by this theorem are smooth up to the boundary (see $[\mathbf{1}$, 2a]). In fact the theory in $[\mathbf{1}$ or $\mathbf{2 b}]$ says that $p(t, \cdot) \in h^{2+\theta}$. A careful computation shows that

$$
V_{\xi \xi}=\frac{\varsigma_{+}-\zeta_{-}}{2} \cos (\xi) \cdot p+\left(\frac{\zeta_{+}-\zeta_{-}}{2} \sin \xi\right)^{2} \cdot q
$$

and $q=p \cdot p_{y} / y$.

If $p \in h^{2+\theta}$, then the first term is a $C^{1}$ function of $\xi$. Except for a constant factor the second term may be written as

$$
(\sin \xi)^{2} q=\left(\frac{\sin \xi}{\xi}\right)^{2} \cdot \frac{\xi^{2}}{y} \cdot p \cdot p_{y}
$$

Since $\left(\xi^{2} / y\right)_{y}=2(\xi / y) \cdot \xi_{y}-(\xi / y)^{2}$, the expression $\xi^{2} / y$ is a $C^{1}$ function of $y$ or $\xi$. Hence $p \in h^{2+\theta}$ implies that $V_{\xi \xi}$ is a $C^{1}$ function of $\xi$. This was the a priori bound on $V_{\xi \xi}$ we needed to prove global analyticity. The only thing we still have to do is get an a priori bound on $\|p\|_{h^{\theta}}$.

There are two ways of obtaining a bound for $\|p\|_{h^{\theta}}$.

The first is to use a result of Aronson and Vazquez [4] which states that after the waiting time $q=v_{x x}$ is a bounded function (up to the boundary). Since $p_{y}=y q / p$, boundedness of $q$ implies that $p$ is $C^{1}$ up to the boundary, and therefore certainly $h^{\theta}$ for any $\theta \in(0,1)$. 
The other method does not use the result of Aronson and Vazquez, but the continuity result of Caffarelli and Friedman [5]. This result states that $p(t, y)$ is continuous up to $y=0$ (i.e. we have an a priori bound on the modulus of continuity of $p$ ). We may choose $\eta$ so small that for given $\varepsilon>0$ and $T>0$

$$
|p(t, y)-p(t, 0)| \leq \varepsilon|p(t, 0)|
$$

holds for $|y| \leq \eta$ and $0 \leq t \leq T$.

Now note that $r=p_{y}$ satisfies the equation

Therefore

$$
r_{t}=\left(\frac{p^{2}}{2}\left(r_{y}+\frac{m-1}{m-1} \frac{r}{y}\right)\right)_{y} .
$$

$$
\begin{aligned}
\frac{d}{d t} \int_{0}^{\eta} \frac{1}{2} r^{2} d y & =\int_{0}^{\eta} r r_{t} d y \\
& =\left[r \frac{p^{2}}{2}\left(r_{y}+\frac{m+1}{m-1} \frac{r}{y}\right)\right]_{0}^{\eta}-\int_{0}^{\eta} \frac{p^{2}}{2}\left(r_{y}^{2}+\frac{m+1}{m-1} \frac{r r_{y}}{y}\right) d y \\
& \leq C-\int_{0}^{\eta} \frac{p^{2}}{2}\left(r_{y}^{2}+\frac{m+1}{m-1} \frac{r r_{y}}{y}\right) d y
\end{aligned}
$$

Apply the Hardy-type inequality

in order to get

$$
0 \leq \int_{0}^{\eta} \frac{r}{y} r_{y} d y \leq 2 \int_{0}^{\eta}\left(r_{y}\right)^{2} d y
$$

$$
\frac{d}{d t} \int_{0}^{\eta} \frac{r^{2}}{2} d y \leq C-(p(0, t)+O(\varepsilon)) \int_{0}^{\eta} r_{y}^{2} d y \leq C
$$

if $\varepsilon$ is small enough. Thus we find that

$$
\int_{0}^{\eta} r^{2} d y \leq \int_{0}^{\eta} r(0, y)^{2} d y+2 C \cdot t
$$

Therefore $p$ remains bounded in $H^{1}$, and, because of the inclusion $H^{1} \hookrightarrow h^{1 / 2}, p$ remains bounded in $h^{1 / 2}$.

REMARKS. 1. Although the second method makes the proof self-contained (except for the Caffarelli-Friedman result) it has the drawback that we have to assume that $p$ belongs to $H^{1}$ for some $t>0$. The Aronson-Vazquez result guarantees that for any initial value of the porous media equation $v_{x x}$ eventually becomes bounded.

2. Our local analysis of the free boundary also applies for $t \in\left(t_{-}, \infty\right)$ or $t \in$ $\left(t_{+}, \infty\right)$, where $t_{ \pm}$are the waiting times of the free boundaries. Thus we recover the results of Aronson and Vazquez [4], and Höllig and Kreiss [6]:

THEOREM. On $\left(t_{+}, \infty\right) \zeta_{+}$is $C^{\infty}$.

By contrast analyticity seems to be a global property (at least in our approach), and we cannot improve upon Theorem 1.

ACKNOWLEDGMENT. I would like to thank P. Clément for urging me to read the work of Da Prato and Grisvard. Also I would like to thank both L. A. Peletier and M. Bertsch for their encouragement and advice. Many lengthy discussions with the latter have had a great influence on the present work.

The referee has pointed out many printing errors in the original version of the manuscript, for which I am grateful. 


\section{BIBLIOGRAPHY}

1. H. Amann, Quasilinear evolution equations and parabolic systems, Trans. Amer. Math. Soc. 293 (1986), 191-227.

2a. S. B. Angenent, Local existence and regularity for a class of degenerate parabolic equations, Math. Ann. (to appear).

2b. _ Abstract parabolic initial value problems, preprint.

3. D. G. Aronson, Nonlinear diffusion problems, Free Boundary Problems: Theory and Applications, vol. I, Pitman, 1983.

4. D. G. Aronson and J. L. Vazquez, Eventual regularity and asymptotic concavity for flows in one dimensional porous media (in preparation).

5. L. A. Caffarelli and A. Friedman, Regularity of the free boundary for the one-dimensional flow of gas in a porous medium, Amer. J. Math. 101 (1979), 1193-1218.

6. K. Höllig and H. O. Kreiss, $C^{\infty}$-regularity for the porous medium equation, Univ. of WisconsinMadison, Computer Sciences Dept., Technical report \#600.

7. G. Da Prato and P. Grisvard, Equations d'évolution abstraites non linéaire de type parabolique, Ann. Mat. Pura Appl. 120 (1979), 329-396.

Department of MAThematics, University of Leiden, P. O. BoX 9512, 2300 RA LEIDEN, THE NETHERLANDS

Current address: Department of Mathematics, University of Wisconsin, Van Vleck Hall, 480 Lincoln Drive, Madison, Wisconsin 53706 